\title{
Some key technics of drop tower experiment device of National Microgravity Laboratory (China) (NMLC)
}

\author{
ZHANG Xiaoqian ${ }^{1}$, YUAN Longgen ${ }^{1}$, WU Wendong ${ }^{1}$, TIAN Lanqiao ${ }^{2}$ \\ \& YAO Kangzhuang ${ }^{2}$ \\ 1. Institute of Engineering Thermophysics, Chinese Academy of Sciences, Beijing 100080, China; \\ 2. Institute of Mechanics, Chinese Academy of Sciences, Beijing 100080, China \\ Correspondence should be addressed to Zhang Xiaoqian (email: zxq@mail.etp.ac.cn) \\ Received December 29, 2004
}

\begin{abstract}
Drop tower is an important ground based facility for microgravity science experiment. The technical performances of the drop tower NMLC are advanced compared with similar facilities in the US, Germany and Japan. The main components such as drop capsule, deceleration devices, release mechanism present its advantages and creativities.
\end{abstract}

Keywords: drop tower facility, drop capsule, deceleration devices, release mechanism.

DOI: 10.1360/102004-21

Microgravity science and application project is a very important part of Chinese space effort. This area has been very active along with the international space activities in $\mathbf{e}-$ cent years and has got many important results. The completion and running of the international space station will further promote the research activities in the area of microgravity science and application.

The National Microgravity Laboratory (China) (NMLC) was established in the middle of the 1990s. Drop tower is one of the main experiment facilities of NMLC. Fig. 1 shows its outlook view. It is $116 \mathrm{~m}$ in height and is located in the southeast of Zhongguancun central area in Beijing. It will be undertaking heavy experiment research in

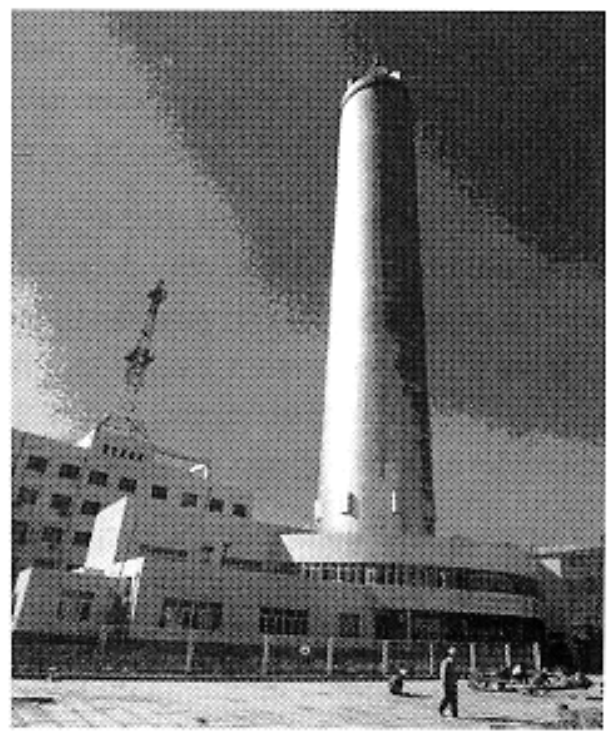

Fig. 1. Drop tower NMLC.

Copyright by Science in China Press 2005 
microgravity science.

In addition to meeting the needs of Chinese space projects, the drop tower of NMLC, which will be open to the world, also has to meet a high requirement in technology. However, it has a budget of construction much lower than its likes in such countries as the US, Germany and Japan. So, we need to establish our own characteristics in constructing the drop tower based on the international experiences and lessons. The main components, such as drop capsule, deceleration devices and release mechanism will be introduced briefly in the present paper.

\section{An overview of the main microgravity drop tower/well experiment facilities in the world}

There are different kinds of facilities such as drop towers/wells, airplanes, sounding rockets, skylab, shuttle and spacelab, and space station etc. (Fig. 2) ${ }^{[1]}$ which can be used to perform microgravity experiments. Each of these facilities has its own characteristics and usage. All these facilities form a complete system to perform different kinds of microgravity experiments. Although the duration of microgravity is relatively short, the drop tower/well facility has many obvious advantages, such as excellent microgravity level, low cost, many test drops per day, ability to use precise equipments, and easy artificial interference etc. All these advantages make it the most important facility for performing microgravity experiments.

In the early 1960s NASA (USA) constructed a drop tower and a drop well at Lewis Research Center (LeRC) ${ }^{[1-3]}$. The drop well is a large-scale facility called "Zero-Gravity Facility". It is $155 \mathrm{~m}$ deep (underground). The duration of microgravity is $5.18 \mathrm{~s}$. The microgravity level is $10^{-5}-10^{-6} \mathrm{~g}_{\mathrm{o}}$. The average deceleration level is $35 \mathrm{~g}_{\mathrm{o}}$ and the peak deceleration level is $65 \mathrm{~g}_{0}$. The drop tower is a simple facility. It is $30.5 \mathrm{~m}$ tall. The duration of microgravity is $2.2 \mathrm{~s}$ and the microgravity level is $10^{-4} \mathrm{~g}_{\mathrm{o}}$. The deceleration level is about $15-30 \mathrm{~g}_{0}$. This drop tower is the most heavily used at LeRC. It routinely supports over 1000 test drops per year (the routine daily schedule allows up to 12 drops).

Japan constructed two microgravity facilities by the end of 1980s and early 1990s. One is the biggest microgravity drop well facility (JAMIC) ${ }^{[4,5]}$ in the world. It is $710 \mathrm{~m}$ deep (underground). It can provide $10 \mathrm{~s}$ duration of microgravity and $10^{-5} \mathrm{~g}_{\mathrm{o}}$ microgravity level. The deceleration level is less than $10 \mathrm{~g}_{0}$. The other microgravity drop well facility $^{[6,7]}$ is $150 \mathrm{~m}$ deep (underground). Its duration of microgravity is $4.5 \mathrm{~s}$. The microgravity level is $10^{-5} \mathrm{~g}_{\mathrm{o}}$ and the deceleration level is also less than $10 \mathrm{~g}_{\mathrm{o}}$.

Germany also constructed a drop tower ${ }^{[8,9]}$ in Bremen by the end of 1980s and early 1990s. It is $146 \mathrm{~m}$ tall. The duration of microgravity is $4.7 \mathrm{~s}$. The microgravity level is $10^{-5}-10^{-6} \mathrm{~g}_{\mathrm{o}}$. The average deceleration level is $25 \mathrm{~g}_{\mathrm{o}}$ and the peak deceleration level is $50 \mathrm{go}$.

It is reported that Russia also constructed a large-scale microgravity facility, but no 
formal materials were published. In addition, there are several small-scale microgravity facilities with duration of microgravity less than $1 \mathrm{~s}$ at institutes and universities in USA.

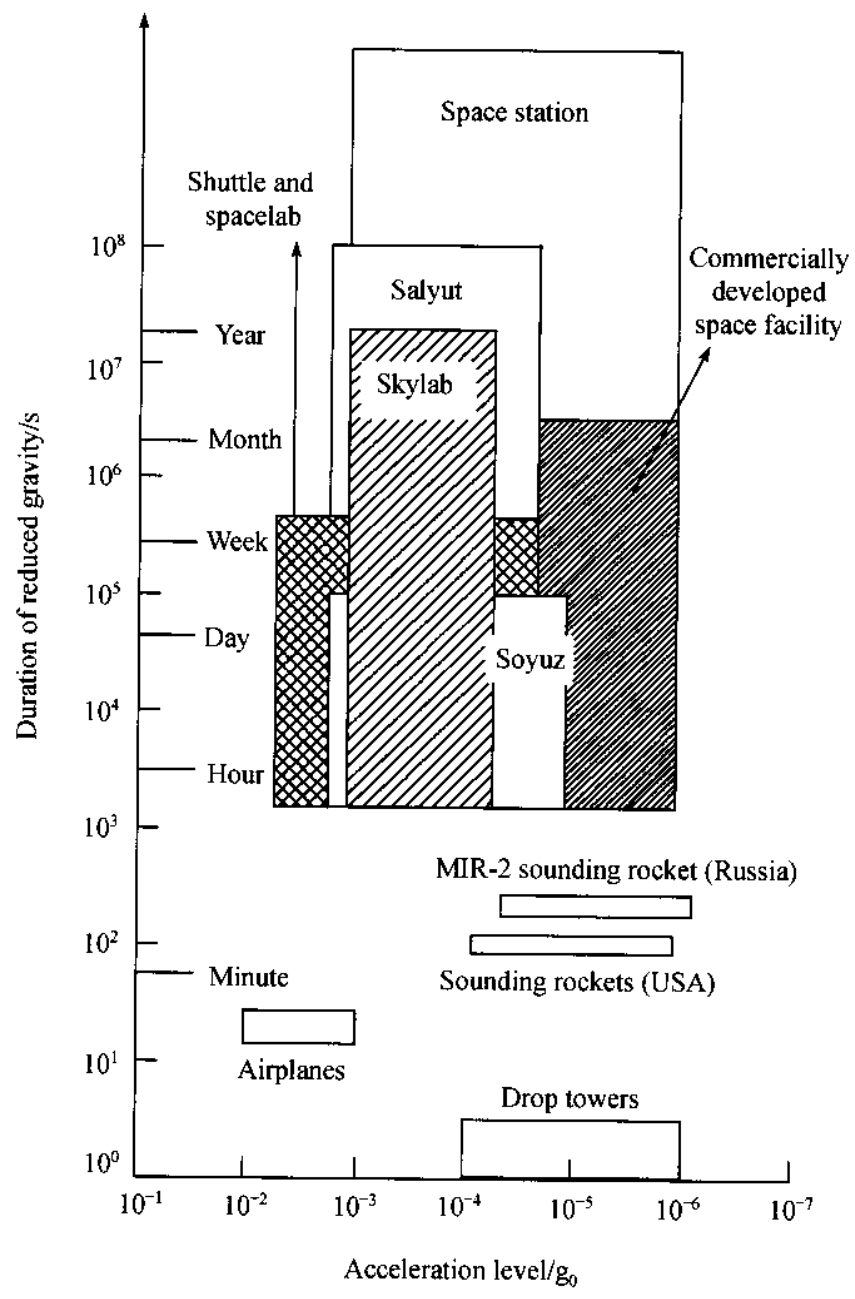

Fig. 2. Characteristic times and acceleration levels of reduced-gravity laboratory facilities. ${ }^{*} \mathrm{~g}_{0}$, local gravitational acceleration.

\section{Drop tower facility NMLC}

The main technical characteristics of the drop tower facility NMLC are:

Duration of microgravity: $3.5 \mathrm{~s}$

Microgravity level: $10^{-5} \mathrm{~g}_{\mathrm{o}}$

Deceleration level: approximately semi-sine-wave

$$
\begin{array}{ll}
\text { average } & \sim 8 \mathrm{~g}_{\mathrm{o}} \\
\text { peak } & \sim 12 \mathrm{~g}_{\mathrm{o}}
\end{array}
$$


This facility consists of drop capsule, deceleration devices, release mechanism, control system, data acquisition system and auxiliary devices. The present paper will only introduce briefly the drop capsule, deceleration devices and release mechanism.

\subsection{Drop capsule assembly}

The schematic drawing of the drop capsule assembly is shown in Fig. 3. The drop capsule assembly consists of inner capsule (experiment package) and outer capsule (drag shield). The space between inner capsule and outer capsule is evacuated. The outer capsule (drag shield) is dropped under normal atmospheric conditions. An axisymmetric aerodynamic contour with low drag coefficient was designed to reduce the air drag during the free fall. The drop capsule assembly is subjected to a big shock when it is decelerated. According to the requirements of the master design the outer capsule should be designed as a light, thin and pressure-resistant shell assembly structure. The strength, rigidity and buckling are guaranteed by detailed mechanical analysis. The drop capsule assembly satisfies the user's requirements completely by qualification tests.

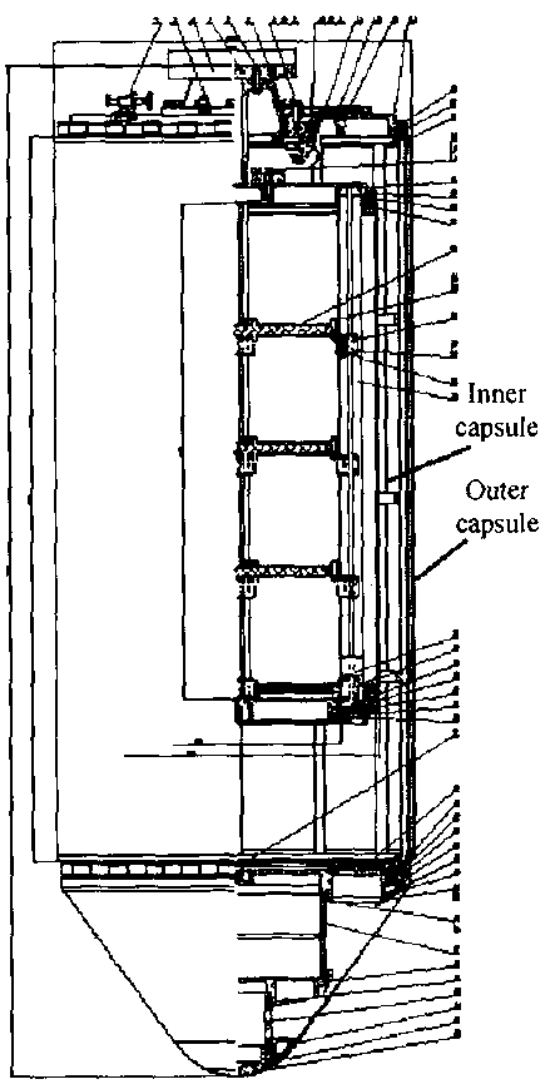

Fig. 3. Schematic drawing of drop caspule assembly.
The inner capsule (experiment package) falls freely within the outer capsule (drag shield). The relative velocity between inner and outer capsule is very small $(<1$ $\mathrm{m} / \mathrm{s})$. Because of both the vacuum environment and small relative velocity, the air drag subjected to inner capsule is very small. So, the microgravity level of the experiment package can reach $10^{-5} \mathrm{~g}_{o}$ or even better.

This light dual-capsule structure with vacuum space between the inner and outer capsule is first used in the world. As compared with similar capsules abroad this drop capsule assembly has the following advantages:

1) In order to reach $10^{-5}-10^{-6} \mathrm{~g}_{\mathrm{o}} \mathrm{mi}$ crogravity level, the air drag subjected to the experiment package must be small enough during the free falling. The air drag subjected to the experiment package during the free falling can be expressed as $F=\frac{1}{2} c_{x} s \rho u^{2}$, where $c_{x}$ is the drag coef-

ficient, $s$ is the effective cross-section, $\rho$ is the air density, and $u$ is the relative velocity. 
When the aerodynamic contour and the structure of the outer capsule were designed, the drag coefficient $c_{x}$ and the effective cross-section $s$ were then decided. In order to get the air drag as small as possible one can reduce the air density and/or reduce the relative velocity. Theoretically, reducing relative velocity will be much more efficient than $\mathbf{e}$ ducing air density. But from the point of view of technology reducing air density will be much easier than reducing relative velocity.

Internationally, both the Bremen (Germany) drop tower and the NASA (USA) LeRC "Zero-Gravity Facility" choose to reduce the air density (single drop capsule free falls within vacuum chamber). The inner diameter and height of the vacuum chamber of Bremen drop tower are $3.5 \mathrm{~m}$ and $110 \mathrm{~m}$ respectively. The total volume (vacuum chamber and decelerating space) need to be evacuated is $1700 \mathrm{~m}^{3} .18$ vacuum pump units with the rate of $3200 \mathrm{~m}^{3} / \mathrm{h}(9000 \mathrm{~L} / \mathrm{s})$ were arranged. The vacuum of the vacuum chamber can be $1 \mathrm{~Pa}$ after all these vacuum pump units worked $2.5 \mathrm{~h}$. Then the microgravity level of the single drop capsule can reach $10^{-5}-10^{-6} \mathrm{~g}_{\mathrm{o}}$ when it falls freely within the $1 \mathrm{~Pa}$ vacuum environment. The inner diameter and height of the vacuum chamber of NASA LeRC “Zero-Gravity Facility” are $6.1 \mathrm{~m}$ and $145 \mathrm{~m}$ respectively. Much more powerful vacuum pump units were arranged. Both reducing air density and relative velocity are chosen in the drop tower NMLC. Then the air drag is reduced very effectively. The volume between the inner and outer capsule need to be evacuated is only $1 \mathrm{~m}^{3}$. A small-scale vacuum pump unit with rate $8 \mathrm{~L} / \mathrm{s}$ is arranged. The required vacuum can be reached in approximately $20 \mathrm{~min}$. The construction cost and the operation and maintenance expenses are reduced greatly by using the present drop capsule assembly.

2) The drop capsule is single capsule structure in both Bremen (Germany) drop tower and NASA LeRC "Zero-Gravity Facility". The speed of the single capsule is accelerated with $1 \mathrm{~g}_{\mathrm{o}}$. At the end of the freefall the speed of the drop capsule can reach approximately $\sim 46$ and $\sim 51 \mathrm{~m} / \mathrm{s}$ respectively. Since the air drag subjected to the drop capsule is proportional to the square of relative velocity, in order to get $10^{-5}-10^{-6} \mathrm{~g}_{\mathrm{o}}$ microgravity level, the vacuum of the vacuum chamber must be evacuated to $1-10 \mathrm{~Pa}$.

For the present facility, since the relative velocity is less than $1 \mathrm{~m} / \mathrm{s}$, the air drag subjected to the inner capsule is also very small. Numerical analysis shows that the vacuum of the space between the inner and outer capsule is less than $1000 \mathrm{~Pa}$ and the microgravity level of the inner capsule can reach $10^{-5} \mathrm{~g}_{\mathrm{o}}$. The data measured by using acceleration meter proves this result. For the present facility the vacuum of the space between inner and outer capsule is less than $30 \mathrm{~Pa}$. Numerical analysis shows that the microgravity level of the inner capsule can reach $10^{-6} \mathrm{~g}_{0}$.

3) The drop capsule assembly of the JAMIC (Japan) drop well is also dual-drop capsule. The vacuum of the space between inner and outer capsule is $10 \mathrm{~Pa}$. But it is a heavy structure. The total mass of the capsule is $5000 \mathrm{~kg}$. For the present facility the total mass of the capsule is only $600-630 \mathrm{~kg}$ since it is a light structure. 
4) The drop capsule assembly of the NASA LeRC 2.2 Second Drop Tower is also dual-drop capsule. But the space between inner and outer capsule is not evacuated. So, it is much simpler in mechanical design and release mechanism. The microgravity level of the inner capsule is only $10^{-4} \mathrm{~g}_{\mathrm{o}}$.

Although the present drop capsule assembly has many advantages mentioned above, it is very difficult in technology. Probably, this is the reason why Bremen (Germany) drop tower and NASA LeRC "Zero-Gravity Facility" choose the very expensive plansingle capsule drops within the vacuum chamber-after detailed analyses and comparison. The present plan indeed met a series of difficulties in technology. All these difficulties have been solved with great efforts.

The first is the falling attitude of the inner and outer capsule. This difficulty is closely related to the release mechanism. It will be discussed later in the part of release mechanism.

The second is the choice of the radial space between the inner and outer capsule. There must be enough space to install the experiment devices, data acquisition system, etc. Meanwhile, the effective cross-section of the outer capsule should be as small as possible in order to reduce the air drag as much as possible. Since it is impossible to eliminate completely the disturbances which will lead to the outer capsule inclined, it will result in the unsafe trouble during the deceleration process. Therefore there must be acceptable space between the inner and outer capsule to ensure the safe problem and the smallest air drag. The drop attitude of the outer capsule has been recorded by using high-speed video camera (1000 frames per second) and the drop attitude of inner capsule relative to the outer capsule has also been recorded by the CCD camera installed on the inner top side of the outer capsule. Now both the drop attitude of the inner and outer capsule are controlled very effectively after a series of technical measures were taken to eliminate the disturbances. The present radial space between the inner and outer capsule is $10 \mathrm{~cm}$. The operation results show that this space is acceptable.

The third is the choice of the axial space between the inner and outer capsule. When the drop capsule assembly falls, the speed of the outer capsule is relatively slow due to the air drag. Meanwhile the speed of the inner capsule is relatively fast since the inner capsule falls in the vacuum environment inside the outer capsule. So, the axial space between the inner and outer capsule must be long enough to ensure the inner capsule will not touch the bottom of the outer capsule before the end of the free falling. In addition, concerning the deceleration process this axial space would not be too long since the deceleration process requires that the inner capsule touches the bottom of the outer capsule just at the end of the free falling, then the inner capsule and the outer capsule as a complete set enters the deceleration process. In this way, the free fall distance can be fully used, and then the facility can have the desired microgravity test duration and also can avoid the extra shock. Step by step increasing the axial space was used in the adjustment process. The results show that this way is safe and effective. The present axial 
space is $81 \mathrm{~cm}$.

The fourth is the control of eccentricity between the centre of mass of the inner and outer capsule and its geometric axis. If the centre of mass is not at the geometric axis, this eccentricity will introduce an initial disturbance at the instant of releasing the capsule which will result in the capsule inclined during the free falling. So, the eccentricity must be controlled precisely. The eccentricity of the Bremen single drop capsule is required to be less than $1 \mathrm{~kg} \cdot \mathrm{m}^{[8]}$. But this requirement in the NMLC duel-drop capsule case is required much more precise, it must be controlled to be less than $0.3 \mathrm{~kg} \cdot \mathrm{m}$. The operation results show that this precise control of the eccentricity is necessary.

The fifth is that the location of the centre of mass of the outer capsule should be as close as possible to the bottom of the capsule. This will ensure that the combination of the resultant forces of air drag and the gravity force of the capsule will recover its right falling attitude of the capsule.

The sixth is that when the inner capsule just touches the bottom of the outer capsule and the assembly of the inner and outer capsule as a whole enters the deceleration process, the inner capsule must sit stably on the bottom of the outer capsule. Otherwise it might result in trouble in the deceleration process. A locking device is installed on the bottom of outer capsule in the present facility. The inner capsule will be caught immediately when it touches the bottom of the outer capsule so that it cannot vibrate.

\subsection{Deceleration devices}

A unique elastic controllable deceleration device was used in NMLC facility. It consists of a three-connector reversible mechanic/electric energy transducer (STS), steel cable, steel ring, strengthened string bag, strengthened elastic rubber strings assembly, energy dissipation resistance box, controlling unit of computer, and emergency deceleration container of small pellets of expanded polystyrene, etc. (Fig. 4). The working process and principle are described as follows:

1) The STS (motor function) elevates the steel ring, rubber strings assembly and bag to predetermined height. The whole assembly stays there and waits for order.

2) The whole assembly starts to fall due to the self weight by the order of the computer $1 \mathrm{~s}$ before the drop capsule assembly falls into the bag. Meanwhile the STS (generator function) is pulled by the bag assembly and generates electricity dissipated by the resistance box. In this way the STS has already rotated when the drop capsule assembly touches the bag for reducing the shock that the STS is subjected to.

3) When the drop capsule assembly with $600-630 \mathrm{~kg}$ and $\sim 34 \mathrm{~m} / \mathrm{s}$ speed drops into the bag assembly, the drop capsule assembly decelerates, and the bag assembly accelerates. Meanwhile the rubber strings assembly is stretching to transduce the kinetic energy of the drop capsule assembly into elastic potential energy stored temporarily. In the meantime the STS speeds up as pulled by the fast falling bag assembly and part of the 
kinetic energy of the drop capsule assembly transduces to electric energy dissipated by the resistance box.

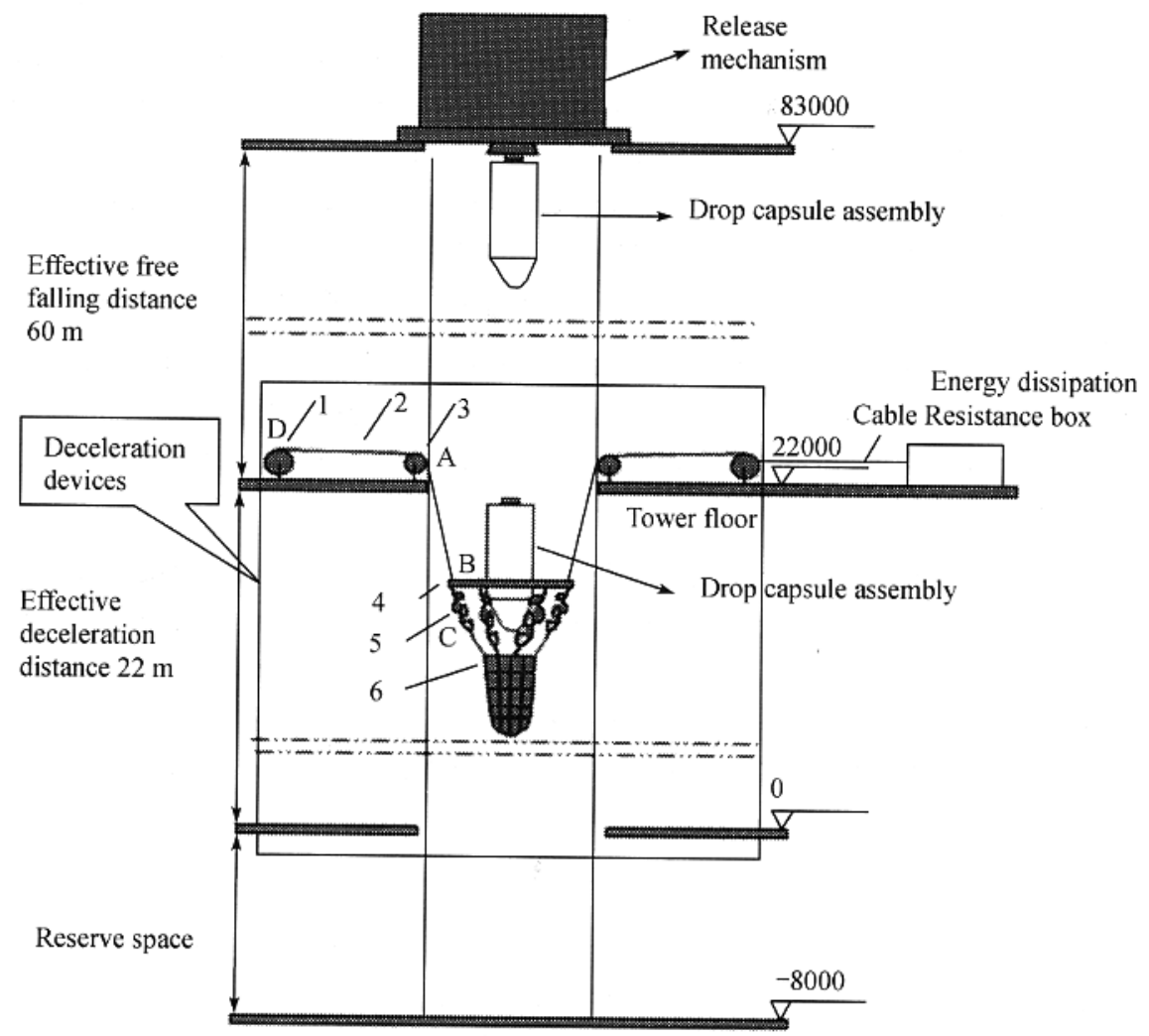

Fig. 4. Sketch of deceleration devices. 1, STS; 2 , steel cable; 3 , guiding wheel; 4 , steel ring; 5 , strengthened elastic rubber strings assembly; 6 , strengthened string bag.

4) When the converted speed of the STS is higher than the falling speed of the bag assembly, the rubber strings assembly starts to shrink and release the stored elastic potential energy. Meanwhile the drop capsule assembly further decelerates.

5) When the falling speed of the drop capsule assembly decelerates to zero, the drop capsule assembly is in static state temporarily viewed from the coordinate fixed on the ground. Meanwhile the bag assembly still falls and the STS still rotates with relatively high speed.

6) The drop capsule assembly free-falls again. Meanwhile the STS transduces its own kinetic energy and the kinetic energy of falling bag assembly and drop capsule assembly into electric energy dissipated by the resistance box. The STS decelerates further.

7) The drop capsule assembly is again in static state.

8) The deceleration devices are at brake state by the order of the computer. Then the drop capsule assembly and bag assembly fall very smoothly and slowly. Finally they Copyright by Science in China Press 2005 
stop at the end place. The deceleration process completely finished. The whole deceleration process can be seen clearly from the curve measured by using the accelerometer (Fig. 5).

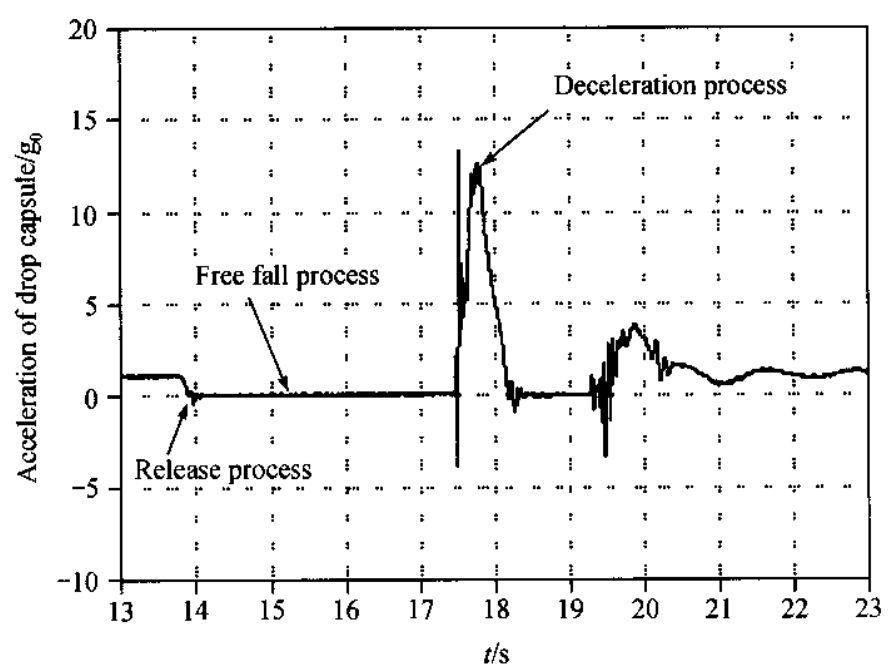

Fig. 5. Measured deceleration curve.

The elasticity coefficient of the rubber strings, the length of the rubber strings assembly and the resistance of the resistance box are the three independent parameters. The primary values of these three independent parameters were chosen by the numerical analysis. Since the rubber strings assembly consists of 96 strings and each rubber string consists of 250 single strings, the elastic resistance subjected to the drop capsule assembly is adjustable by reasonably matching the numbers of the rubber string, strings and the length of the rubber strings. Theoretically the resistance of the resistance box could change continually to meet the different states of the deceleration process. In fact it is found during the adjustment process that as long as the step by step changing resistance are well coupled with the rubber strings assembly this deceleration device can satisfy the requirements of the global property. The most important technical problem which must be solved completely is that "upward moving" of the drop capsule assembly is not allowed during the deceleration process, that is, the falling speed of the drop capsule assembly slows down from $\sim 34 \mathrm{~m} / \mathrm{s}$ to $0 \mathrm{~m} / \mathrm{s}$, then the upward moving speed is not allowed. If the coupling between the elasticity coefficient of the rubber strings assembly, the length of the rubber strings assembly and the resistance of the resistance box is not suitable the drop capsule assembly might be shot upward by the rubber strings assembly and this probably will result in trouble in the deceleration process. In the adjustment process for the sake of safety the three independent parameters were chosen firstly based on the numerical analysis, then the drop distance $\Delta H$ varies gradually from $1,3,5,8,10$ and $15 \mathrm{~m}$ until $64 \mathrm{~m}$. Each $\Delta H$ has a corresponding group of the three parameters. Finally the "upward moving" problem is well solved. The present acceptable total mass of the drop capsule assembly ranges from $600 \mathrm{kgs}$ to $630 \mathrm{kgs}$. 
The present deceleration devices have the following advantages obviously in comparison with similar devices abroad.

1) The deceleration device of NASA LeRC "Zero-gravity Facility" is a big container with $3.6 \mathrm{~m}$ diameter and $6.1 \mathrm{~m}$ depth filled with small pellets of expanded polystyrene. The friction between the drop capsule and the small pellets of expanded polystyrene decelerates the drop capsule. The average deceleration rate is $35 \mathrm{~g}_{\mathrm{o}}$ and the peak is $65 \mathrm{~g}_{0}$. A similar device is also used in German Bremen drop tower facility. The container is $1.6 \mathrm{~m}$ in diameter and $10 \mathrm{~m}$ in height, filled with 10 tons small pellets of expanded polystyrene. The average deceleration level is $25 \mathrm{~g}_{\mathrm{o}}$ and the peak is less than $50 \mathrm{~g}$. These deceleration devices cannot be controlled actively.

In the early time (from the 1960s to the early 1990s) the deceleration device of NASA LeRC 2.2 Second drop tower was a $2.2 \mathrm{~m}$ deep sand pit and the drag shield has three deceleration spikes attached to the bottom ${ }^{[10]}$. When the spikes impact the sand pit the drag shield decelerates. The deceleration level is about $40-100 \mathrm{~g}_{0}$. Later on by the middle of the 1990s this device was improved. The present deceleration device is an air bag $^{[3]}$. When the drag shield assembly impacts the air bag the drop is terminated. The deceleration level is greatly improved. The peak values of the present deceleration level are 15 to $30 \mathrm{~g}_{\mathrm{o}}$. This deceleration process also cannot be controlled actively.

For the present elastic controllable deceleration technique, as long as the elasticity coefficient of the rubber strings assembly, the length of the rubber strings assembly and the resistance of the resistance box are well coupled, the deceleration process can be controlled actively by the computer. The performance of the present deceleration devices is close to a semi-sine-wave with average deceleration $\sim 8 \mathrm{~g}_{\mathrm{o}}$ and peak deceleration $\sim 12$ $\mathrm{g}_{\mathrm{o}}$. Obviously the performance of the present deceleration devices is better than the small pellets of expanded polystyrene technique and the air bag technique.

2) Although the performance of the present deceleration technique is comparable to the JAMIC deceleration technique, the construction cost of the present deceleration devices is much lower than the JAMIC deceleration devices.

In summary, the characteristics of the deceleration devices of NMLC drop tower are advanced in technique and unique.

\subsection{Release mechanism}

The electromagnetic release mechanism is used in the present facility. Since the drop capsule assembly is a dual-capsule with vacuum space between the inner and outer capsule, the inner capsule and outer capsule are well sealed. The electric-pneumatic combination release mechanism used in the single drop capsule of German Bremen drop tower and the shearing bolt release mechanism used in the single drop capsule of NASA LeRC "Zero-gravity Facility" cannot be used in the present facility. The highly stressed music wire release mechanism used in NASA LeRC 2.2 Second drop tower also cannot be used in the present facility, since the space between the experiment package and the drag 
shield is not evacuated.

This electromagnetic release mechanism consists of assembly platform at 83-m height, electromagnetic attraction assembly, top components of outer capsule, top components of inner capsule, mechanical lock/release mechanism of outer capsule, mechanical lock/release mechanism of inner capsule, control panel on the ground, control panel within centre control room, control system, and hanging basket operation platform at 83-m height. Two key technical problems must be solved for the release mechanism: the initial disturbance problem, and the initial attitude of drop capsule.

Since the principle of the present release mechanism is electromagnetic attraction /release without mechanical connection, the "force" connection between electromagnetic attraction assembly and top components of outer and inner capsule is not "point" connection any more, but "plane" connection. The release mechanism of German Bremen drop tower single drop capsule, NASA LeRC "Zero-Gravity Facility" single drop capsule and NASA LeRC 2.2 second drop tower dual-capsule is "point" connection. The present "plane" connection makes the problems even more difficult to solve. Because of the machining accuracy, the assembling accuracy, the accuracy of the mass balance device and a variety of the experiment devices it is very difficult to ensure:

a) The centre of mass of the electromagnetic attraction assembly, the outer capsule and the inner capsule assembly is exactly located at their own geometric central line respectively.

b) When the drop capsule assembly is suspended and is waiting for the release order, the centre of mass of the electromagnetic attraction assembly and the centre of mass of the drop capsule assembly are on the same vertical line.

c) The attraction force of the electromagnetic attracting assembly for the drop capsule assembly gets through the centre of mass of the electromagnetic attraction assembly.

So, during the release process the release mechanism might introduce an initial "force" disturbance which will affect the free falling attitude of the inner and outer capsule. Although there are many technical difficulties mentioned above, the electromagnetic release mechanism has a very attractive advantage which can greatly reduce the initial "force" disturbance. Reasonably matching the parameters of the electromagnetic release mechanism, the attraction force is reducing gradually when the electricity was shut down suddenly. The attraction force is still active within a certain distance. This gradually varying release process makes the change from normal gravity to microgravity of the experiment devices not a sudden changing process. Then the initial disturbance is greatly reduced.

A comprehensive technique was chosen during the adjustment process. The connecting deviation of electromagnetic attraction assembly and drop capsule assembly is controlled very accurately to be less than $0.25 \mathrm{~mm}$ and a $0.15 \mathrm{~mm}$ disconnecting space at the key location of the force connecting line between the electromagnetic attraction as- 
sembly and the drop capsule assembly. This technique ensures the release mechanism works perfectly, the falling attitude of the drop capsule assembly is vertical and the deceleration process works safely.

\section{Current state and prospects}

The microgravity pool boiling heat transfer experiments conducted in the NMLC drop tower have got pictures of stable microgravity boiling and heat transfer data. These results show that when the overheating is relatively low, the microgravity subcooled nucleate boiling heat transfer efficiency is $10 \%$ higher than the normal gravity situation. While the overheating is relatively high the microgravity subcooled dual-film boiling heat transfer efficiency is $20 \%$ lower than the normal gravity situation. The behavior of bubble/film changes a lot. Further experiments and detailed analysis on the experiment data are in progress. Part of the results was published in International Symposium on Physical Science in Space/Spacebound in Toronto, Canada in May, 2004. These experiments are the first step for preparing the next step orbit experiment.

The microgravity laminar dust flame propagation experiments and the flame-flow interaction experiments in microgravity will be arranged in the near future.

Two test drops can be arranged daily. If necessary the routine daily schedule allows up to 4 drops.

The NMLC drop tower facility is in regular run. Many important projects in microgravity science and application are conduced in this facility. This facility is also a "window" to arrange the international cooperation in the area of microgravity science and application. This facility also demonstrates NMLC's ability in the area of microgravity science and application.

\section{References}

1. Lekan, J., Neumann, E. S., Sotos, R. G., Capabilities and constraints of NASA's ground-based reduced gravity facilities, in Second International Microgravity Combustion Workshop, 1993, NASA CP-10113.

2. Lekan, J., Microgravity research in NASA ground-based facilities, AIAA Paper, 1989, 89-0236.

3. LeKan, J. et al., Users Guide for the 2.2 Second Drop Tower of the NASA Lewis Research Center, 1996, NASA TM 107090.

4. JAMIC Japan Microgravity Center, 20, Nishi2-chome, kita-ku, Sapporo 060, Hokkaido, Japan, 1997.

5. Japan Microgravity Center User's Guide Japan Microgravity Center (JAMIC), Japan Space Utilization Promotion Center (JSUP), 1991.

6. Micro-Gravity Laboratory of Japan (MGLAB), 1990.

7. Users Guide for the Drop Experiment Facility, Micro-Gravity Laboratory of Japan, MG-Technical Report$002,1995$.

8. Drop Tower "Bremen” User Manual, Germany, Version 2.2, 1992.

9. ZARM Drop Tower Bremen User Manual, Version 28, 2000.

10. John, B., Haggard, Jr., Cochran, T. H., Hydrogen and hydrocarbon diffusion flames in a weightless environment, NASA TN D-7165, 1973. 